\title{
Factoring digital health for improved outcomes in management of Non Communicable Diseases
}

\author{
Neera Gupta, Tushar Dixit ${ }^{1}$, Vikram Reddy K. ${ }^{2}$ \\ Head Medical Education, Novo Nordisk Education Foundation, Bangalore, ${ }^{1}$ Head, Information Technology, Novo Nordisk, ${ }^{2}$ Medical Advisor, \\ Novo Nordisk Education Foundation Bangalore, India
}

\section{A B S T R A C T}

Make in India is a flagship campaign from the Government of India which is aimed at various sectors. Indian health-care system can take advantages by introducing digital technologies to health care. The possible uses of health information technology as a method to engage with people involving communicable and noncommunicable diseases (NCDs) are wide-ranging. Health information technology can support interventions for prevention, diagnosis, managing surveillance, disease monitoring, and treatment compliance of many conditions. Digital health can offer various technologies such as wearables that are tracked by various mobile or smartphone applications to facilitates patient engagement, self-monitoring, and implementing behavior changes in NCDs such as diabetes. By incorporating the interactive audiovisual items, the digital health tools can create greater interest and engage people from different geographies, age, gender, and culture. Currently, the common digital heath tech issues include hesitancy from doctors to adopt any new product or technology, difficulty in reaching and accessing the needy patients, cultural diversity in population, and inadequate infrastructure. The ethical issues of consent, data security, and privacy of patients need to be addressed as they are highly sensitive in nature.

Key words: Digital Health, telemedicine, Non Communicable Diseases

\section{INTRODUCTION}

Digital health is the need of the hour today. With changing environment and the advent of new government in India, the "role of technology in healthcare sector" is gaining significant momentum. Make in India is one such example of flagship campaign from the Government of India which is aimed at various sectors. Noncommunicable diseases (NCDs) such as cancer, cardiovascular diseases and stroke, chronic respiratory diseases, and diabetes are the leading causes of morbidity and mortality worldwide. They are largely preventable if risk factors such as sedentary lifestyle, physical inactivity, unhealthy dietary habits, smoking, and use of alcohol are managed. The regulatory environment

\begin{tabular}{|l|l|}
\hline \multicolumn{2}{|c|}{ Access this article online } \\
\hline Quick Response Code: & Website: \\
\hline & www.josh.net \\
\hline & \\
\hline
\end{tabular}

for medical technology is ambiguous and complex in India and factors such as affordability, accessibility, availability, and awareness, add to its complexity. With new government, manifesto plan is to create a robust platform for integrating mobile phones to National eHealth Authority and technology driven health-care delivery. The health-care systems in India may benefit more when world-class medical devices are manufactured indigenously. By introducing digital health technologies to society broadly, quality and affordable of primary health care can be improved. ${ }^{[1]}$

\footnotetext{
This is an open access article distributed under the terms of the Creative Commons Attribution-NonCommercial-ShareAlike 3.0 License, which allows others to remix, tweak, and build upon the work non-commercially, as long as the author is credited and the new creations are licensed under the identical terms.
}

For reprints contact: reprints@medknow.com

How to cite this article: Gupta N, Dixit T, Reddy VK. Factoring digital health for improved outcomes in management of Non Communicable Diseases. J Soc Health Diabetes 2016;4:102-7. 


\section{Revolution in Digital Health}

Digital health can directly address the issue of health-care accessibility by bringing online features through internet and smartphone applications (apps) for medical professionals and patients. Digital tools having customer relationship management can offer online consultation, integrate wellness, and prevention programs. ${ }^{[2]}$

Digital health currently offers technology such as wearable that are tracked by various mobile or smartphone apps which supports patient engagement. ${ }^{[3,4]}$ With the help of digital health patients can self-monitor and behavior changes can be encouraged by examining the data. ${ }^{[5]}$

The urban modern society is increasingly getting familiar with digital health technologies and makes a variety of transactions. The wireless technology and remote monitoring devices offer various opportunities that include remote video consultation, sensor technology, treatment adherence, etc. ${ }^{[6]}$

Digital health stands for the transfer of health resources and healthcare by electronic means. ${ }^{[7]}$ It covers the major areas such as the delivery of health information by internet and telecommunications use information technology and e-commerce to improve public health services. ${ }^{[6]}$ Medical and public health practice supported by mobile devices, such as mobile phones, patient-monitoring devices, personal digital assistants, and other wireless devices. ${ }^{[8]}$ This involves the use of voice and short messaging service (SMS) as well as more complex functionalities and applications including General Packet Radio Service, third-and fourth-generation mobile telecommunications (3G and $4 \mathrm{G}$ systems), global positioning system, and Bluetooth technology. ${ }^{[6]}$

Digital health should not be viewed as a distinct modality of care, but rather as a tool to bridge the gaps in existing care delivery systems and an opportunity to enhance them. Where it is important to place emphasis on, that digital health offers the advantage of increasing access to underserved populations, especially in rural areas. ${ }^{[9]}$

The intensification of various technologies and medicine has created a disruption in health care and the general pharmaceutical sector which have lately been recognized. Using suitable digital applications, the drug development field can be benefited with the help of newer technologies..$^{[10]}$

To reduce inequalities among people with different levels of health literacy, the digital interventions should be accessible and acceptable to target population. By incorporating the interactive audiovisual items, the digital health tools can create interest and engage people from different geography, age, gender, and culture..$^{[1]}$

It will be easier for patients suffering from diabetes to track their levels of blood glucose and other data pertaining to the amount of carbohydrate intake, medicines administered, and physical activities using any validated digital health tool. These tools can help the treating physician and other health-care professionals for guidance and support. ${ }^{[12,13]}$ It is recommended that the person-based approach is critical for the identification of interventions for designing a digital health tool. This helps the digital tool designers in understanding and accommodating the perspectives of target users of digital interventions and makes it more user-friendly. ${ }^{[14]}$

The areas where digital health has made some impact are the health and medical websites and platforms, the telemedicine, and telehealth tools. These digital health means have improved ways to deliver public health and its monitoring. ${ }^{[15]}$ In digital marketing and digital health social networks, it is observed that $90 \%$ of the actors only observe, and they do not participate, $9 \%$ may contribute sparingly, and $1 \%$ of actors actually create the most of the new contents. This is famous as $1 \%$ rule and $90 \%, 9 \%$, and $1 \%$ actors are also described as lurkers, contributors, and super users, respectively. ${ }^{[16]}$ The social networks and online activities can influence a person's daily lives by motivating them to monitor their lifestyles, diet, and exercise. ${ }^{[17]}$

One intriguing direction for research in relation to the concept of the digitally engaged patient is that which pursues an investigation into the embodied and affective dimension of digital technology usage. As noted above, patients often invest the medical encounter with emotion, particularly if they are suffering and desperate for help. When the medical encounter is mediated via digital media devices, emphasis may be invested in the devices themselves and the social relationships that are established via the devices between users and their health-care providers or with other patients. A digitally engaged patient like any other patient may go through heavy emotions while suffering and may have desperation for help. These medical issues should be mediated by digital health devices appropriately. ${ }^{[18]}$

\section{Role of TeCHNOLOgY IN HEALTH-CARE SYSTEMS}

The possible uses of digital health technology as a method to engage with people involving communicable and 
NCDs are wide-ranging. Health information technology can support interventions for prevention, diagnosis, managing surveillance, disease monitoring, and treatment compliance of many conditions. Telemedicine is expected to be bridging the rural-urban gap.

\section{PREVENTION}

International Telecommunication Union is the United Nations specialized agency for information and communication technologies and the World Health Organization, have come together in a ground breaking new partnership to focus on the use of mobile technology to improve NCDs prevention and treatment. NCDs are largely preventable through tackling common risk factors: Tobacco use, unhealthy diet, physical inactivity, and the harmful use of alcohol. ${ }^{[19]}$ The mobile phones and other mobile communication technologies can connect people anytime and anywhere. The transmission of text messages (SMS), photographs, and data happen at the touch of a button. These advances have led to the development of mobile health (mHealth). Mobile phones have been leveraged for client health education using text messaging to address risk factors for NCDs in developed countries. ${ }^{[20]}$ Mobile (m) solutions will be primarily SMS or apps based and will include a range of services including $\mathrm{mAwareness,}$ mTraining, mBehavioral change, mSurveillance, mTreatment, mDisease management, and mScreening building on existing successful pilots and scaling them to a wide population level. ${ }^{[21]}$

The World Health Professions Alliance supported by the International Federation of Pharmaceutical Manufacturers and Associations has launched an app to help individuals and their health professionals reduce the risk of NCDs. The app is called "Healthy Score" which helps individuals rate their habits with a traffic light system. Four biometric indicators and four behavior indicators provide an overview of the person's health status. The app is free to download for Android and iOS devices. ${ }^{[21]}$

The four biometrics indicators monitored are body mass index and serum cholesterol to reduce the risk of cardiovascular diseases, fasting blood sugar to help prevent diabetes, and blood pressure to check for hypertension..$^{[22]}$

Similarly, the lifestyle indicators include the avoidance of tobacco use and harmful alcohol consumption, improving diet, undertaking regular sufficient physical activity, achievement, and maintenance of healthy weight, manage tension and stress, promote preventive care, and screening for preventable illnesses. ${ }^{[22]}$
A traffic light system helps people to rate their habits in terms of healthiness. Behaviors in red are risky; those in yellow indicate caution and those in green are healthy. The app also provides users with customizable reminders to help them move their behaviors toward green. ${ }^{[23]}$

\section{DIAGNOSIS}

Mobile diagnostic kits can help in preventive healthcare. Access to advanced diagnostic technologies can help in identifying diabetes, cancer, or even cardiovascular diseases. ${ }^{[24,25]}$

In Madhya Pradesh and Gujarat, GE Healthcare has set up diagnostic centers at select government medical colleges. The charges for the patients are unchanged, the center and the equipment will be run by the company and its partners. ${ }^{[26]}$

Access to diagnosis and treatment services for neglected tropical diseases is a challenge, particularly in areas with extreme poverty and poor infrastructure. Rapid diagnostics tests for diseases that are prevalent will beneficial in resource-poor settings. ${ }^{[27]}$

\section{MONITORING}

Remote health-care/self-monitoring devices that help identify and validate symptoms, and technology that enables doctor-to-doctor interaction, are going to be the way forward. ${ }^{[28]}$ The concept of Self-monitoring blood glucose (SMBG) is still not well-known in India as compared to the West. In India, a meager $0.3 \%$ of diabetics use SMBG as opposed to almost 22\% in developed countries. Lack of awareness regarding the complication of diabetes and benefits of regular monitoring are key factors for this. Remote monitoring for cardiac devices is also feasible for patients implanted with cardiac rhythm management (CRM) devices. ${ }^{[26]}$

Through telemedicine, high-quality medical services can be brought to wherever patients are rather than transporting patients to distant and expensive tertiary care centers. Through cost-effective video conferencing expertise could be transferred to suburban areas. This will help to improve healthcare in rural areas. ${ }^{[29]}$

\section{TREATMENT ADHERENCE}

Regular care and informational support are helpful in improving disease-related health outcomes. Communication technologies can help in providing such care and support. The cell phone voice and short message 
services can help improvement in health outcomes and care processes involving both patients and health-care providers. It was reported that there were significant improvements noted in compliance with medicine taking, asthma symptoms, $\mathrm{HbA} 1 \mathrm{C}$, stress levels, smoking quit rates, and self-efficacy. ${ }^{[30]}$

It has been reported that text message reminders had increased health workers' adherence to malaria treatment guidelines with improvements in program, and patient management. ${ }^{[31]}$

\section{DEADDICTION}

The addiction treatment field offers a promising setting for telemedicine use. The chronic nature of addiction disorders calls for methods for clinicians to stay connected with patients over extended periods of time. Face-to-face contacts between patients and clinicians are limited to scheduled appointments or group sessions. Counselors are not available when they're most needed: Outside the treatment setting, where patients make decisions to stay sober or not. Telemedicine extends the providers' availability and offers patients an immediate resource. ${ }^{[32]}$

As part of its smoking prevention campaign new smartphone app is launched that offers interactive support from friend or partner. The SmokeFree Buddy App is one of the such smartphone app introduced in Switzerland. ${ }^{[33]}$

\section{Digital HeAlth ANd Diabetes}

In NCDs, diabetes is one therapeutic area where technology is integral part of diagnosis, monitoring, and treatment. The use of digital health can help people with diabetes save time and money and improve health-care systems for better society. There are various digital platforms either in use or in development phase such as Glooko, for example, allows patients to download blood glucose readings to their smartphones, integrate food, and lifestyle data then share that information with health providers. Diabetes Pilot records glucose, insulin, and other measurements; tracks food nutrients; includes a food database, and Glucose Buddy, for iOS, lets users record meals, exercise, insulin levels, and shares records with physicians.

GoCap, a pen cap that tracks insulin and wirelessly connects with a smartphone, a replacement cap for insulin pens, the GoCap reads insulin amounts and times, then communicates via Bluetooth with a phone or connected glucometer.
Once big, bulky machines, glucose monitors are now as sleek as an iPhone. Vendors such as Gmate integrate an app and smart meter that connects to the iPhone headphone jack, then measures a patient's blood glucose reading.

Smart contact lens project, "built to measure glucose levels in tears via a tiny wireless chip and miniaturized glucose sensor embedded between two layers of soft contact lens material." Merging this technology with an app can make glucose measurements available to both wearer and their doctor.

Engineers from Boston University created a closed-loop bionic pancreas system that uses continuous glucose monitoring and subcutaneous delivery of rapid-acting insulin and glucagon as directed by an algorithm. ${ }^{[34]}$

The components of diabetes self-management education are healthy eating, SMBG, medication adherence, and diabetes complications risk reduction behavior. There are several mobile apps available to help patients with diabetes to self-manage the disease. ${ }^{[35]}$

\section{CURRENT STATUS IN INDIA}

With the development of Systematic Medical Appraisal Referral and Treatment (SMART) cities (cities where information technology is the principal infrastructure and the basis for providing essential services to residents along with many technological platforms including automated sensor networks and data centers) and SMART health, India is also pacing up with the inclusion of digitalization in most of the sectors. Currently, in India, some aspects of telehealth are being implemented at the clinic-to-hospital level, wherein general practitioners connect with specialists for the second opinion. Remote health-care/self-monitoring devices that help identify and validate symptoms are getting established. "SMART Health India" has been developed by George Institute researchers in India, Australia, and the UK for this purpose. It is a unique low-cost, high-quality health-care delivery to provide state-of-the-art health-care solutions for NCDs. It utilizes advanced mHealth technologies and helps health-care professionals in taking individualized decisions for the SMART of individual members of the community. ${ }^{[36]}$ With internet penetration increasing in India, hospitals have started looking at online tools as a viable option. For example, the All India Institute of Medical Sciences has recently launched its preregistration portal for patient's appointment and admission. ${ }^{[25]}$ As a step toward the further enhancement of changing diabetes 
in children program, the group has launched e-registry platform for children with type 1 diabetes in India. This national registry will help in improving the quality and consistency of diabetes care for children and provide the scientific community a vital tool for proper surveillance, future planning, and implementation of necessary actions even by policy makers. ${ }^{[37]}$

\section{ChAllenges AND Future}

It can be used to facilitate data collection and to encourage health-care consumers to adopt healthy lifestyles or to self-manage chronic conditions. It can also be used to improve health-care service delivery processes by targeting health-care providers or communication between these providers and their patients. Hence, for example, mobile technologies can be used to provide clinical management support in settings where there are no specialist clinicians, and they can be used to send patients test results and timely reminders of appointments. ${ }^{[38]}$

Despite the many benefits of digital mobile apps, there are some challenges as there is no guarantee that the apps will be used systematically. Hence, if the apps become more well-known, it is necessary to promote its systematic use among the health services. Simply making this tool available is not enough. Further research is needed to evaluate the tool's applicability and acceptance and to measure the impact of this type of initiative on eHealth, with a view to improving health practices, empowering users, and ultimately, achieving better clinical outcomes. ${ }^{[39]}$

\section{SCIENTIFIC AUTHENTICITY}

The vast majority of healthcare apps on the market have not been tested to a level that would be considered acceptable by medical researchers. The traditional "gold-standard" practices of medical research are not feasible for these dynamic, fast-paced apps. The formally constituted, broad-based, placebo-controlled, peer-reviewed medical research takes $5 \frac{1}{2}$ years is regarded as quite a long duration. The focus of these apps is on rapid delivery rather than proof of effect. ${ }^{[39]}$

\section{PRIVACY AND DATA PROTECTION}

It is important to ensure public trust while maintaining confidentiality and privacy of personal health data. The ethical issues of consent, data security, and privacy need to be addressed as they are often highly sensitive in nature. ${ }^{[6]}$ Apps with potentially harmful content were also to be identified and regulated. The regulators should insist on provide comprehensive, evidence-based support. Second, checking the maker's privacy policy is critical. If client data is protected in a robust and unambiguous manner, it is a good indication that the app developer is at least ethical even if it is not effective. ${ }^{[40]}$ There are already heavy requirements in the US and EU where an App is the same as a medical device which is subject to validation and patient safety based requirements.

\section{CONCLUSION}

We have already stepped in the digital era with increasing potential and use of digital platforms in all sectors, especially in health-care systems. Digital health is a need for continuously changing the environment and rapid advances in the field of medicine. Health-care providers should actively and appropriately select the digital health-care solutions to extend the reach of disease self-management to wider patient's communities, provide for the individualized care, and in time information. The success of medical technological innovation can further be backed by powerful financial incentives, a supportive regulatory system, and fostering an environment for quality research.

\section{Acknowledgments}

The authors thank Croissance ${ }^{\mathrm{TM}}$ Clinical Research Group, Hyderabad, India for providing medical writing assistance in the development of this manuscript.

Financial support and sponsorship

Nil.

\section{Conflicts of interest}

There are no conflicts of interest.

\section{REFERENCES}

1. Ramana TV. Make in India: Is a possible reality project or merely an illusion? Int J Acad Res 2015;2:10-20.

2. Garewal K. HIT, Insightful Coverage of Healthcare Technology. 10 Indian Digital Health Startups to Watch; 29 October, 2015. Available from: http://www.hitconsultant.net/2015/10/29/1019-10 -indian-digital-health-startups-to-watch/. [Last accessed on 2016 Jun 15].

3. Frist WH. Connected health and the rise of the patient-consumer. Health Aff (Millwood) 2014;33:191-3.

4. SteinhubI SR, Muse ED, Topol EJ. Can mobile health technologies transform health care? JAMA 2013;310:2395-6.

5. Birnbaum F, Lewis D, Rosen RK, Ranney ML. Patient engagement and the design of digital health. Acad Emerg Med 2015;22:754-6.

6. Hollis C, Morriss R, Martin J, Amani S, Cotton R, Denis M, et al. Technological innovations in mental healthcare: Harnessing the digital revolution. Br J Psychiatry 2015;206:263-5.

7. WHO. Global Observatory for e-Health, http://www.who.int/goe/ 
Gupta, et al.: Digital health in management of Non Communicable Diseases

en/. 2013. [Last accessed 2016 May 10].

8. World Health Organization. mHealth New Horizons for Health Through Mobile Technologies. Global Observatory for eHealth Series. Vol. 3. Bangalore: World Health Organization; 2013.

9. Agboola SO, Bates DW, Kvedar JC. Digital Health and Patient Safety. JAMA 2016;315:1697-8.

10. Hird N, Ghosh S, Kitano H. Digital health revolution: Perfect storm or perfect opportunity for pharmaceutical R\&D? Drug Discov Today 2016;21:900-11.

11. Rowsell A, Muller I, Murray E, Little P, Byrne CD, Ganahl K, et al. Views of people with high and low levels of health literacy about a digital intervention to promote physical activity for diabetes: A qualitative study in five countries. J Med Internet Res 2015; 17:e230.

12. Salber P, Niksch A. Digital health tools for diabetes. J Ambul Care Manage 2015;38:196-9.

13. Buntz B. Digital health startup focuses on diabetes prevention. Medical Device and Diagnostic Industry. 2012;34(12).

14. Yardley L, Morrison L, Bradbury K, Muller I. The person-based approach to intervention development: Application to digital health-related behavior change interventions. J Med Internet Res 2015; $17:$ e30.

15. Lupton D. Critical perspectives on digital health technologies. Sociol Compass 2014;8:1344-59.

16. van Mierlo T. The $1 \%$ rule in four digital health social networks: An observational study. J Med Internet Res 2014;16:e33.

17. Ba S, Wang L. Digital health communities: The effect of their motivation mechanisms. Decis Support Syst 2013;55:941-7.

18. Lupton $\mathrm{D}$. The digitally engaged patient: Self-monitoring and self-care in the digital health era. Soc Theory Health 2013;11:256.

19. ITU-WHO Mobile Health for Non-Communicable Diseases (NCDs) Initiative. Available from: http://www.itu.int/en/ITU-D/ ICT-Applications/Pages/Be_Healthy_intro.aspx. [Last accessed on 2016 Jun 10].

20. Cole-Lewis $\mathrm{H}$, Kershaw T. Text messaging as a tool for behavior change in disease prevention and management. Epidemiol Rev 2010;32:56-69.

21. The Editor. App for reducing risk of non-communicable diseases. Pharmaceutical J 2014; DOI: 10.1211/PJ.2014.20067098.

22. What is the WHPA Health Improvement Card? Available from: http://www.healthyscore.org/. [Last accessed on 2016 Jun 10].

23. Help Patients Make Heathy Choices with WHPA App. Available from: http://www.fip.org/www/index.php?page=news_publicati ons\&news $=$ newsitem\&newsitem $=182$. [Last accessed on 2016 Jun 25].

24. Reforming Healthcare in India Through Technology; 2015. Available from: http://www.financialexpress.com/healthcare/it-healthcare/ reforming-healthcare-in-india-through-technology/41537/. [Last accessed on 2016 May 25].

25. Gopal Ravi. Reforming Healthcare in India Through Technology. Express Healthcare: Feb 11 2015. http://www.financialexpress. com/healthcare/it-healthcare/reforming-healthcare-in-indiathrough-technology/41537/.

26. Medical Technology Industry in India Riding the Growth
Curve. Available from: http://www. 2.deloitte.com/content/ dam/Deloitte/in/Documents/life-sciences-health-care/ in-Ishc-medical-technology-in-India-noexp.pdf. [Last accessed on 2016 Jul 25].

27. A New Mobile App is Hoping to Ease Diagnosis of Neglected Diseases in Africa; 06 June, 2016. Available from: http://www. iafrikan.com/2016/06/06/a-new-mobile-app-is-hoping-to-easediagnosis-of-neglected-diseases-in-africa/. [Last accessed on 2016 Jul 25].

28. Healthcare Tech in India Needs a New Life: Experts Mamta Bhardwaj Bengaluru, DHNS; 02 January, 2016. Available from: http://www.deccanherald.com/content/520739/ healthcare-tech-india-needs-life.html. [Last accessed on 2016 Jun 30].

29. Telemedicine Alone Can Bridge Divide in Urban-Rural Healthcare, Says Expert. Available from: http://www.health.economictimes. indiatimes.com/news/health-it/telemedicine -alone-can-bridgedivide-in-urban-rural-healthcare-says-expert/52815320. [Last accessed on 2016 Jul 10].

30. Krishna S, Boren SA, Balas EA. Healthcare via cell phones: A systematic review. Telemed J E Health 2009;15:231-40.

31. Zurovac D, Sudoi RK, Akhwale WS, Ndiritu M, Hamer DH, Rowe AK, et al. The effect of mobile phone text-message reminders on Kenyan health workers' adherence to malaria treatment guidelines: A cluster randomised trial. Lancet 2011;378:795-803.

32. Molfenter T, Boyle M, Holloway D, Zwick J. Trends in telemedicine use in addiction treatment. Addict Sci Clin Pract 2015;10:14.

33. Better with Two: New App for Giving Up Smoking. Available from: http://www.spectra-online.ch/en/spectra/topics/ better-with-two-new-app-for-giving-up-smoking-454-10.html. [Last accessed on 2016 Jun 28].

34. Source. Available from: http://www.informationweek.com/ healthcare/patient. [Last accessed on $2016 \mathrm{Jul}$ 30].

35. Shah VN, Garg SK. Managing diabetes in the digital age. Clin Diabetes Endocrinol 2015;1:16.

36. The George Institute for Global Health, India. http://www. georgeinstitute.org.in/sites/default/files/smart-health-indiabrochure.pdf. (Last accessed 2016 Jul 19].

37. Kumar KM, Saboo B, Rao PV, Sarda A, Viswanathan V, Kalra S, et al. Type 1 diabetes: Awareness, management and challenges: Current scenario in India. Indian J Endocrinol Metab 2015;19 Suppl 1:S6-8.

38. Free C, Phillips G, Watson L, Galli L, Felix L, Edwards P, et al. The effectiveness of mobile-health technologies to improve health care service delivery processes: A systematic review and meta-analysis. PLoS Med 2013;10:e1001363.

39. Ordúñez P, Tajer C. Disseminating cardiovascular disease risk assessment with a PAHO mobile app: A public eHealth intervention. Rev Panam Salud Publica 2015;38:82-5.

40. Medical Apps: Lifesavers or Dangers to Health? Available from: http://www.smh.com.au/technology/smartphone-apps/ medical-apps-lifesavers-or-dangers-to-health-20160502-gokkzi. html. [Last accessed on 2016 Jun 30]. 